\title{
$D J-1$ affects oxidative stress and pyroptosis in hippocampal neurons of Alzheimer's disease mouse model by regulating the Nrf2 pathway
}

\author{
LIN CHENG and WEI ZHANG
}

Zhang Zhongjing College of Chinese Medicine, Nanyang Institute of Technology, Nanyang, Henan 473004, P.R. China

Received August 26, 2019; Accepted March 5, 2020

DOI: $10.3892 /$ etm.2021.9989

\begin{abstract}
Studies have confirmed that $D J-1$ is associated with diseases associated with the nervous system, including Alzheimer's disease (AD). However, the role of $D J-1$ in the pathogenesis of AD has not been clarified. To investigate the effect of $D J-1$ on brain tissue damage and cognitive function in AD mice and its possible mechanism, 5XFAD transgenic mice were used as AD model mice and $D J-1$ in the brain was overexpressed by transfection of a lentiviral containing a specific targeting $D J-1$ gene into the bilateral hippocampus of mice. Following lentivirus infection, the Morris water maze test was performed to assess the cognitive function of the mice. When the behavioral evaluation was completed, the brain tissue of the mouse was examined. Pathological changes were observed by hematoxylin-eosin staining. The levels of relevant indicators were analyzed by reverse transcription-quantitative PCR, ELISA and western blotting. Bilateral hippocampal injection of a lentivirus containing $D J-1$ significantly increased the expression of $D J-1$ in the hippocampus of 5XFAD transgenic mice. Overexpression of $D J-1$ in the brain could ameliorate brain tissue damage, $\beta$-amyloid protein $(\mathrm{A} \beta)$ deposition and cognitive function in 5XFAD mice. Compared with the sham group, the reactive oxygen species activity and malondialdehyde content in the brain tissue of $D J-1$ overexpressing 5XFAD mice were
\end{abstract}

Correspondence to: Dr Lin Cheng, Zhang Zhongjing College of Chinese Medicine, Nanyang Institute of Technology, 80 Changjiang Road, Nanyang, Henan 473004, P.R. China

E-mail: lincheng_12@163.com

Abbreviations: AD, Alzheimer's disease; $\mathrm{A} \beta, \beta$-amyloid protein; HE, hematoxylin-eosin staining; IL-18, interleukin-18; IL-1 $\beta$, interleukin-1 $\beta$; MDA, malondialdehyde; NLRP3, NLR pyrin domain containing 3; Nrf2, nuclear factor erythroid 2-related factor 2; ROS, reactive oxygen species; SOD, superoxide dismutases; TUNEL, terminal transferase-mediated biotin dUTP nick end labeling; TXNIP, thioredoxin-interacting protein

Key words: Alzheimer's disease, oxidative stress, pyroptosis, Parkinson disease protein 7, nuclear factor erythroid 2-related factor 2, nucleotide-binding domain, leucine-rich repeat pyrin domain containing 3 significantly decreased, while the superoxide dismutase activity was significantly increased $(\mathrm{P}<0.05)$. In addition, $D J-1$ overexpression decreased the expression of caspase- 1 and the levels of interleukin (IL) $1 \beta$ and IL-18 in the hippocampus while ameliorating the death of hippocampal neurons in 5XFAD mice and without affecting the expression of caspase-3. Overexpression of $D J-1$ resulted in a significant decrease in nuclear factor erythroid 2-related factor 2 (Nrf2) protein expression in the cytoplasmic while significantly increasing the expression of Nrf2 in the nucleus. Simultaneously, $D J-1$ overexpression in the brain inhibited the activation of nanoparticles activate the NLR pyrin domain containing 3 inflammatory bodies in brain tissue. Overexpression of DJ-1 in the brain could repair brain tissue damage, $A \beta$ deposition and cognitive function in 5XFAD mice, and its mechanism may be associated with an inhibition of oxidative stress and neuronal pyroptosis by regulating the Nrf2 signaling pathway.

\section{Introduction}

Alzheimer's disease (AD) is a common neurodegenerative disease that is characterized by cognitive dysfunction (1). AD has become the fourth most common lethal human disease following cardiovascular disease, cancer and stroke (2). In-depth study of the pathogenesis of AD has become one of the main foci of neuroscience research.

In the variety of hypotheses concerning $\mathrm{AD}$, oxidative stress has received increasing attention. Oxidative stress is a state in which an intracellular oxidative-antioxidative imbalance leads to stress damage in the organism. It has been confirmed that oxidative stress is directly associated with the formation of conditions such as senescence (3), Parkinson's disease (4) and $\mathrm{AD}$ (5). The development of drugs targeting antioxidant stress to treat $\mathrm{AD}$ has become a promising area of research.

Recent studies have shown that pyroptosis is closely associated with the pathogenesis of AD (6). Pyroptosis is a programmed cell death that is mediated by caspase-1 (7). Cell swelling and rupture while releasing pro-inflammatory factors leads to cell death. Several studies have revealed molecular signaling pathways associated with pyroptosis, such as the nuclear factor erythroid 2-related factor 2 (Nrf2) pathway (8) and the thioredoxin-interacting protein (TXNIP) pathway (9), among which Nrf2 is also considered to be a necessary factor for the regulation of nanoparticles activate the NLR pyrin domain 
containing 3 (NLRP3) inflammasome (10). NLRP3 inflammasome activation is the most important complex protein formed during pryoptosis, which is considered to be a key factor mediating the development of neuroinflammation (11).

The $D J-1$ gene was first reported in 1997 by Nagakubo et al (12) in Japan and the human $D J-1$ gene was mapped to $1 \mathrm{p} 36$. A number of studies have shown that $D J-1$ is associated with diseases associated with the nervous system, such as Parkinson's, Alzheimer's and depression. DJ- 1 also serves a role in a number of physiological processes such as anti-oxidative stress (13) and anti-neuronal apoptosis (14). As an important nuclear transcription factor, Nrf2 is involved in the regulation of the expression of various antioxidant enzymes in cells and serves an important role in the prevention of oxidative stress (15). Studies have shown that $D J-1$ can regulate the expression of Nrf2 signaling pathway in Parkinson's disease and diabetic nephropathy (16-18). DJ-1 inhibits dopaminergic denaturation by activating the Nrf2 signaling pathway in Parkinson's disease (19). Nrf2 can affect the activation of the NLRP3 inflammasome by regulating the TXNIP pathway $(20,21)$. In addition, activation of the NLLRP3 inflammasome further mediates Caspase-1-based pyroptosis (22). Based on these studies, it was hypothesized that $D J-1$ may be involved in the pathogenesis of $\mathrm{AD}$ by activating Nrf2 signaling pathways to regulate oxidative stress and pyroptosis. To verify this hypothesis, 5XFAD transgenic mice were used as AD model mice to overexpress $D J-1$ in mouse brain by lentiviral system transfection to investigate the role of $D J-1 /$ Nrf2 signaling pathway in the pathogenesis of AD.

\section{Materials and methods}

Animals and materials. A total of 60 5XFAD transgenic mice (male, 3 months old, 20-25 g) were provided by Nanyang Institute of Technology. The mice received sterile rodent chow and water ad libitum, were maintained on a $12 \mathrm{~h}$ light/dark cycle in controlled temperature $\left(24 \pm 1^{\circ} \mathrm{C}\right)$ and at $55 \%$ humidity. All experimental procedures were ethically approved by the Animal Use and Care Committee of the Nanyang Institute of Technology (Nanyang, China) and were conducted in accordance with the National Institute for Health 'Guide for the Care and Use of Laboratory Animals' (23).

Lentiviral particles containing a specific targeting $D J-1$ gene and lentiviral particles containing non-specific RNA sequences were constructed and provided by Guangzhou Qingyin Biotechnology Co., Ltd.

A total RNA extraction kit (Tiangen Biotech Co., Ltd.), PCR primers (Shanghai Shenggong Biology Engineering Technology Service, Ltd.), a protein concentration determination kit (Beijing Solarbio Science \& Technology Co., Ltd.) and interleukin (IL)-1 $\beta$ (cat. no. hz-EL-6452), IL-18 (cat. no. hz-EL-M0730c), $\beta$-amyloid protein $(\mathrm{A} \beta)_{40}$ (cat. no. hz-EL-M0067) and $A \beta_{42}$ ELISA kits (cat. no. hz-EL-M0068c; Huzhen Industrial Co., Ltd.) were also used.

Experimental design. To begin, 10 of the 5XFAD transgenic mice were randomly selected to receive the $D J-1$ lentivirus injected into the bilateral hippocampus ( $D J-1$ group) and another 10 mice were injected with lentiviruses of unrelated
RNA sequences in the bilateral hippocampus as the negative control (NC) group. Mice in the sham group had the anterior fontanel exposed without injection of lentivirus. Then, 2 months after the lentivirus infection, the Morris water maze test was performed. After the behavioral evaluation was completed, the mice were anesthetized by intraperitoneal injection of $0.1 \%$ sodium pentobarbital ( $35 \mathrm{mg} / \mathrm{kg}$, Sigma-Aldrich, Merck $\mathrm{KGaA}$ ) and were decapitated to obtain the brain tissue of the mice. Sections of the brain tissues were subjected to hematoxylin-eosin (HE) staining to observe pathological changes; the other tissues were used for reverse transcription-quantitative (RT-q) PCR, ELISA and western blotting.

Intracerebroventricular injections. The mice were placed into a stereotaxic frame (RWD Life Science) following anesthesia. The skin was incised under aseptic conditions and the periosteum of the skull was isolated to reveal the anterior fontanel. The co-ordinates of the anterior fontanel sagittal axis (A), coronal axis (L) and vertical axis (V) were recorded. The Paxinos map was referred to in order to determine the right hippocampal dentate gyrus injection point (A1=A-3.3, L1=L+1.8) (24). The fixed micro-sampler was on the TAXIC-653 brain locator. $D J-1$ $(5 \mu \mathrm{l} ; 10,000 \mathrm{TU} / \mu \mathrm{l})$ containing lentivirus or negative control was injected; the tip of the micro syringe was inserted into the dentate depth $(\mathrm{V} 1=\mathrm{V}+4.4)$ and the virus was slowly injected, following which the needle was removed for $10 \mathrm{~min}$. The left hippocampal dentate gyrus injection point was determined by reference to the Paxinos map (A1=A-3.3, L1=L-1.8) (24) and $D J-1$ or control lentivirus was injected in the same manner. In the sham group, only the periosteum of the skull was isolated and the anterior fontanel was revealed. After the animal exhibited no intracranial hemorrhage, the bone suture was closed with bone wax and the skin sutured. Following intramuscular injection of penicillin, the mouse was returned to its cage.

$R T-q P C R$. After the hippocampal tissue $(100 \mathrm{mg})$ was sufficiently ground, $1 \mathrm{ml}$ TRIzol ${ }^{\circledR}$ (Thermo Fisher Scientific, Inc.) was added for lysis. Subsequently, total RNA was extracted using the phenol-chloroform method (25). Ultraviolet spectrophotometry (Nanodrop ${ }^{\text {TM }}$ 2000; Thermo Fisher Scientific, Inc.) was used to determine the purity of RNA by evaluating the A260/A280 ratio. cDNA was obtained by reverse transcription (RT) using a GoScript ${ }^{\mathrm{TM}}$ Reverse Transcription System (Promega Corporation) from $2 \mu \mathrm{g}$ RNA. Each RT reaction mixture was prepared as follows: RNA template $(5 \mu \mathrm{l})$, Oligo dT primers $(2 \mu \mathrm{l})$, super pure dNTP $(2 \mu \mathrm{l})$ and $\mathrm{H}_{2} \mathrm{O}(5.5 \mu \mathrm{l})$. The reaction conditions involved heating to $70^{\circ} \mathrm{C}$ before rapid cooling on ice for $2 \mathrm{~min}$ at room temperature, following which transient centrifugation was performed at $500 \mathrm{x} \mathrm{g}$ for $30 \mathrm{sec}$ at $4^{\circ} \mathrm{C}$. Subsequently, $4 \mu \mathrm{l} 5 \mathrm{X}$ first-strand buffer, $0.5 \mu \mathrm{l}$ RNasin and $1 \mu \mathrm{l}$ M-MLV were added prior to gentle mixing with a pipette. After being kept at $25^{\circ} \mathrm{C}$ for $10 \mathrm{~min}$, the sample was incubated at $42^{\circ} \mathrm{C}$ for $50 \mathrm{~min}$ before termination at $95^{\circ} \mathrm{C}$ for 5 min. qPCR was subsequently performed using $\mathrm{SYBR}^{\circledR}$ Premix Ex Taq ${ }^{\mathrm{TM}}$ kit according to manufacturer's protocol (Takara Bio, Inc.). The sequences of the primers used were as follows: DJ-1 forward, 5'-UGGAGACGGUCAUCCCUGU-3' and reverse, 5'-ACCUCUGCCAGUAGGGACA-3; $\beta$-actin forward, 5'-ACACTGTGCCCATCTAGGAGG-3' and reverse, 5'-AGGGGCCGGACTCGTCATACT-3'. The thermocycling 
conditions were: Initial denaturation at $94^{\circ} \mathrm{C}$ for $1 \mathrm{~min}$, followed by 35 cycles of $94^{\circ} \mathrm{C}$ for $30 \mathrm{sec}, 55^{\circ} \mathrm{C}$ for $30 \mathrm{sec}$ and $72^{\circ} \mathrm{C}$ for $1 \mathrm{~min}$, a final extension at $72^{\circ} \mathrm{C}$ for $2 \mathrm{~min}$ and hold at $4^{\circ} \mathrm{C}$. $\beta$-actin was used as the internal reference. Data were analyzed using the $2^{-\Delta \Delta \mathrm{Cq}}$ method (26).

Western blotting. The hippocampal tissue samples were lysed by RIPA lysate (Beyotime Institute of Biotechnology) to extract total protein. Nuclear and cytosolic proteins were extracted by a Nuclear/Cytosol Fractionation kit (BioVision, Shanghai, China). Protein concentration was determined using a BCA Protein Assay Kit (Beijing Solarbio Science \& Technology Co., Ltd.). Protein ( 80 or $120 \mu \mathrm{g}$ ) was separated on a denatured $12 \%$ polyacrylamide gel and transferred onto a polyvinylidene fluoride membrane. Membranes were blocked in TBS with $0.1 \%$ Tween-20 containing 5\% non-fat milk and then incubated overnight at $4{ }^{\circ} \mathrm{C}$ with the corresponding primary antibodies. The primary antibodies used were as follows: Anti- $D J-1$ (1:500 dilution; cat. no. SAB4500248; Sigma-Aldrich, Merck KGaA), anti-Nrf2 (1:500 dilution; cat. no. SAB4501984; Sigma-Aldrich, Merck KGaA), anti-NLRP3 (1:500 dilution; cat. no. ERP20425; Abcam), anti-apoptosis-associated speck-like protein containing a CARD (ASC;1:500 dilution; cat. no. SAB4501315; Sigma-Aldrich, Merck KGaA), anti-caspase-1 p10 (1:1,000 dilution; cat. no. AF1681; Beyotime Institute of Biotechnology), anti-cleaved caspase-3 (1:1,000 dilution; cat. no. AF1150; Beyotime Institute of Biotechnology), anti- $\beta$-actin (1:500 dilution; cat no sc-7210; Santa Cruz Biotechnology, Inc.), and anti-histone H3 (1:500 dilution; cat. no. ab176842; Abcam). Membranes were then incubated with horseradish peroxidase-conjugated secondary antibody IgG (1:1,000 dilution; cat. no. 61-1620; Invitrogen, Thermo Fisher Scientific, Inc.) for $1 \mathrm{~h}$ at room temperature. The gels were developed using an enhanced chemiluminescent detection kit (Beyotime Institute of Biotechnology). The relative expression levels of the protein of interest were analyzed using Quantity One gel image analysis software (version 3.0; Thermo Fisher Scientific, Inc.).

$H \& E$ staining. The hippocampus tissue was fixed in $4 \%$ paraformaldehyde solution overnight at room temperature and sectioned $(5 \mu \mathrm{m})$ following paraffin embedding. Paraffin sections were deparaffinized using xylene and rehydrated using a graded alcohol series $(100-70 \% \mathrm{v} / \mathrm{v})$, following which they were then stained with $5 \%$ hematoxylin solution for $15 \mathrm{~min}$ at temperature and counterstained with $0.5 \%$ eosin solution for 5 min prior to dehydration with a graded alcohol series (100-70\% v/v). Following clearing and mounting, the sections were observed using light microscopy (magnification $\mathrm{x} 400$ ).

ELISA. NP-40 buffer (cat. no. P0013F; Beyotime Institute of Biotechnology) was added to the hippocampus tissue. Following sufficient grinding, the tissue homogenate was centrifuged at $5,000 \mathrm{x}$ g for $15 \mathrm{~min}$ at $4^{\circ} \mathrm{C}$ and the supernatant was collected. ELISA was performed according to the manufacturer's instructions for interleukin (IL)- $1 \beta$, IL-18, $A \beta_{40}$ or $\mathrm{A} \beta_{42}$. Finally, the absorbance value at $450 \mathrm{~nm}$ was measured with a microplate reader and the corresponding concentration was calculated.
Morris water maze test. The Morris water maze test was performed as described previously (27). In brief, each training was performed in 4 steps, each step beginning from a new entry point. The mice faced the pool wall prior to entering the water and the time from the entry of the water to the climbing of the platform (latency) was recorded. If the mouse did not find the platform within $90 \mathrm{sec}$, the experimenter guided it onto the platform and recorded it as $90 \mathrm{sec}$. After the mice climbed the platform and rested for $30 \mathrm{sec}$, the next training step began. The average value of 4 training results was recorded as the latency of the day. After 4 days of training, the platform was removed on the 5th day. The mice were allowed to swim for $90 \mathrm{sec}$ in the pool and their trajectory recorded. The percentage of total time and total distance traveled by the mouse in the quadrant of the platform was analyzed.

Terminal transferase-mediated biotin dUTP nick end labeling (TUNEL) assay. Hippocampus tissue was cut into $6 \mu \mathrm{m}$ sections using a Mcllwain microtome (Science Products $\mathrm{GmbH}$ ) and fixed overnight in $4 \%$ paraformaldehyde at $4^{\circ} \mathrm{C}$. In situ detection of hippocampal neuronal apoptosis was performed using the ApopTag Fluorescein in situ Apoptosis Assay kit (EMD Millipore; cat. no. S7110) by modification of genomic DNA using terminal deoxynucleotidyl transferase according to the manufacturer's instructions. Following TUNEL labeling, the nuclei were labeled with DAPI (Abcam), and the neurons labeled with $\mathrm{NeuN}$ and examined in at $\geq 5$ fields under a fluorescence microscope (magnification $\mathrm{x} 200$ ). The number of TUNEL positive cells in the hippocampus was counted.

Oxidative stress marker detection. Fresh hippocampus tissue was removed and rinsed with PBS, homogenized in NP-40 buffer (cat. no. P0013F; Beyotime Institute of Biotechnology) and centrifuged at $12,000 \mathrm{x} \mathrm{g}$ at $4^{\circ}$ for $15 \mathrm{~min}$. Protein quantification was subsequently performed as described previously (28). Reactive oxygen species (ROS) content in the brain tissue was detected by fluorescent probe 2',7'-dichlorofluorescin diacetate (DCFH-DA) method. In brief, $100 \mathrm{mg}$ hippocampal tissue was fully lysed with $300 \mu 1$ lysis buffer at room temperature. After the sample was centrifuged at $12,000 \mathrm{x} \mathrm{g}$ for $10 \mathrm{~min}$ at $4^{\circ} \mathrm{C}$, the supernatant was collected. The sample was placed with $50 \mu 1$ supernatant in a 96-well plate, and $10 \mu \mathrm{l} /$ well DCFH-DA solution was added and incubated in the dark at $37^{\circ} \mathrm{C}$ for $30 \mathrm{~min}$. Samples were measured by using a fluorescence microplate reader (DTX800, Beckman Coulter, Inc.). The content of malondialdehyde (MDA) in brain tissue was detected by the thiobarbituric acid method. In brief, $100 \mathrm{mg}$ of hippocampal tissue was lysed and homogenized with $250 \mu \mathrm{l}$ pre-chilled buffer at $4^{\circ} \mathrm{C}$. The samples were mixed with $10 \mu \mathrm{l}$ butylated hydroxytoluene, $250 \mu 11 \mathrm{M}$ phosphoric acid, $250 \mu \mathrm{l}$ 2-thiobarbiruric acid and incubated at $60^{\circ} \mathrm{C}$ for $1 \mathrm{~h}$. Subsequently, the sample was centrifuged at $12,000 \mathrm{x} \mathrm{g}$ for $5 \mathrm{~min}$ at $4^{\circ} \mathrm{C}$ and the supernatant was collected. The supernatant $(50 \mu \mathrm{l})$ was placed in a 96 -well plate and measured on a microplate reader (BioTek Instruments Inc.). Superoxide dismutase (SOD) activity was determined by the xanthine oxidase method. In brief, hippocampal tissue was homogenized in lysis buffer. The lysate was centrifuged at 12,000 x g for $10 \mathrm{~min}$ at $4^{\circ} \mathrm{C}$ and the supernatant collected. Samples and SOD standards were placed in 96-well plates at $20 \mu \mathrm{l} /$ well and 
$160 \mu \mathrm{l}$ of working reagent was added to each well. Xanthine oxidase enzyme was added at $20 \mu \mathrm{l} /$ well and incubated at $37^{\circ} \mathrm{C}$ for $1 \mathrm{~h}$ in the dark. Measurements were performed using a microplate reader at $450 \mathrm{~nm}$. All operations were performed in strict accordance with the manufacturer's protocols.

Statistical analysis. All statistical analyses were performed using SPSS software (v.16.0; SPSS Inc.). Data are expressed as the mean \pm standard deviation. Comparisons among three or more groups were conducted using a one-way analysis of variance followed by Tukey's post hoc test. $\mathrm{P}<0.05$ was considered to indicate a statistically significant difference.

\section{Results}

Effect of DJ-1 overexpression in brain on cognitive function and brain tissue damage in 5 XFAD trans genic mice. The results of RT-qPCR and western blotting showed that the expression levels of $D J-1 \mathrm{mRNA}$ and protein in the hippocampus of 5XFAD transgenic mice injected with $D J-1$ lentivirus in bilateral hippocampus were significantly increased compared with the sham group $(\mathrm{P}<0.05$; Fig. $1 \mathrm{~A}$ and $\mathrm{B})$. There was no significant difference in the expression of $D J-1 \mathrm{mRNA}$ and protein in hippocampus of 5XFAD mice between the NC and sham groups $(\mathrm{P}>0.05)$.

HE staining was used to observe the pathological changes of hippocampus and neurons (Fig. 1C). It was identified that hippocampal neurons in the sham group were disordered in hippocampus and a large number of necrotic neurons appeared in the hippocampus and the nucleus staining was deep and the nucleus exhibited pyknosis. The above pathological changes were improved in the DJ-1 group.

The deposition of a large amount of $A \beta$ outside the neurons is one of the typical pathological features of AD. The content of $\mathrm{A} \beta_{40}$ and $\mathrm{A} \beta_{42}$ in the hippocampus of $\mathrm{AD}$ mice was analyzed by ELISA. It was identified that $A \beta_{40}$ and $A \beta_{42}$ levels in the $D J-1$ group were significantly decreased compared with the sham group $(\mathrm{P}<0.05$; Fig. 1D), suggesting that $D J-1$ overexpression in brain can ameliorate $\mathrm{A} \beta$ deposition in hippocampus of $\mathrm{AD}$ mice.

The Morris water maze test demonstrated that the latency of finding the platform during the acquired training in the $D J-1$ group was significantly shorter compared with that in the sham group $(\mathrm{P}<0.05$; Fig. $1 \mathrm{E})$. There was no significant difference in the swimming speed of the visual platform test between the $D J-1$ and sham groups $(\mathrm{P}>0.05$; Fig. $1 \mathrm{~F})$. During the exploratory training period, the percentage of total time in the target quadrant (Fig. 1G) and the percentage of the total distance (Fig. $1 \mathrm{H}$ ) of the $D J-1$ group were significantly increased compared with the sham group $(\mathrm{P}<0.05)$. Simultaneously, the number of mice crossing the platform in the $D J-1$ group was significantly increased compared with the sham group $(\mathrm{P}<0.05 ;$ Fig. 1I).

Effect of brain DJ-1 silencing on oxidative stress in 5XFAD transgenic mice. Oxidative stress is an important pathological manifestation of AD. $D J-1$ is considered to be an oxidative stress response protein (29) and is closely associated with the regulation of oxidative stress in nerve cells (30). Therefore, the effect of $D J-1$ overexpression in brain on oxidative stress in brain tissue of 5XFAD mice was analyzed. It was identified that compared with the sham group, the ROS activity and MDA content in the brain tissue of the $D J-1$ group were significantly decreased, while the SOD activity was significantly increased $(\mathrm{P}<0.05$; Fig. 2). There was no significant difference in the content of ROS, MDA and SOD between the sham and NC groups. These results suggested that overexpression of $D J-1$ in the brain may reduce oxidative stress damage in the brain of 5XFAD mice.

Effect of DJ-1 overexpression in brain on neuronal pyroptosis in 5XFAD transgenic mice. Neuronal death is another important feature of AD. The effect of $D J-1$ overexpression in the brain on neuronal cell death in 5XFAD mice was analyzed. The TUNEL assay results showed that the fluorescent spots of cell death primarily corresponded to the distribution of neuronal cell bodies (Fig. 3A). A large number of TUNEL-positive cells were observed in the hippocampus of the sham group, while the number of TUNEL-positive cells in the DJ-1 group was decreased. Quantitative analysis also confirmed that the number of TUNEL-positive cells in the hippocampus of $D J-1$ group was significantly decreased compared with that of the sham group $(\mathrm{P}<0.05$; Fig. 3A). The results suggested that overexpression of $D J-1$ may significantly improve neuronal cell death in 5XFAD mice.

Caspase-1 and caspase- 3 are important molecules regulating cell death; caspase- 3 is an important executive molecule of apoptosis (31) and caspase-1 is mainly involved in the mediation of pyroptosis (32). Therefore, the expression levels of caspase- 1 p10 and cleaved caspase- 3 in hippocampus of 5XFAD mice were analyzed. It was identified that the overexpression of $D J-1$ in the brain significantly decreased the activation of caspase- 1 in the hippocampus of 5XFAD mice without affecting the activation of caspase-3 (Fig. 3B).

Loss of cell membrane integrity and release of inflammatory cell contents to induce inflammatory responses are important features of pyroptosis, unlike apoptosis (33). It was identified that $D J-1$ overexpression significantly decreased the IL-1 $\beta$ and IL-18 levels in the hippocampus tissues of the 5XFAD mice (Fig. 3C). These results revealed that the effect of $D J-1$ overexpression in brain on the death of hippocampal neurons in $\mathrm{AD}$ mice may be associated with the regulation of pyroptosis.

Effect of DJ-1 overexpression in the brain on the expression of Nrf2-NLRP3 axis in the hippocampus of 5XFAD transgenic mice. The Nrf2 pathway, as the most important endogenous antioxidant stress pathway identified at present, serves a key role in the development of AD (34). Simultaneously, Nrf2 activation may be involved in the regulation of pyroptosis by regulating the NLRP3 inflammasome $(10,35)$. In addition, $D J-1$ regulates the expression of the Nrf2 signaling pathway in a variety of diseases $(16,17,20)$. To further investigate the potential mechanism by which $D J-1$ overexpression improves brain tissue damage in 5XFAD mice, the expression of associated proteins in the Nrf2-NLRP3 axis of brain tissue associated with oxidative stress and pyroptosis was analyzed. Western blotting data indicated that $D J-1$ overexpression significantly decreased the expression of $\mathrm{Nrf} 2$ protein in 
A

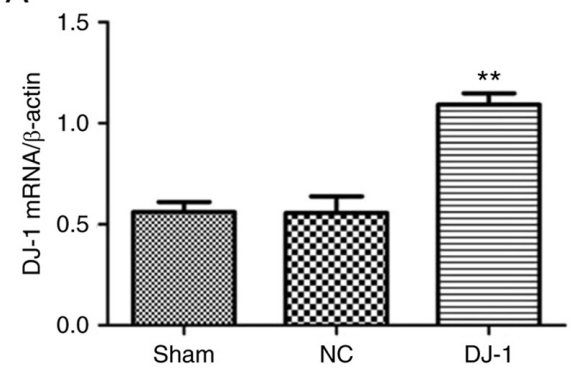

B

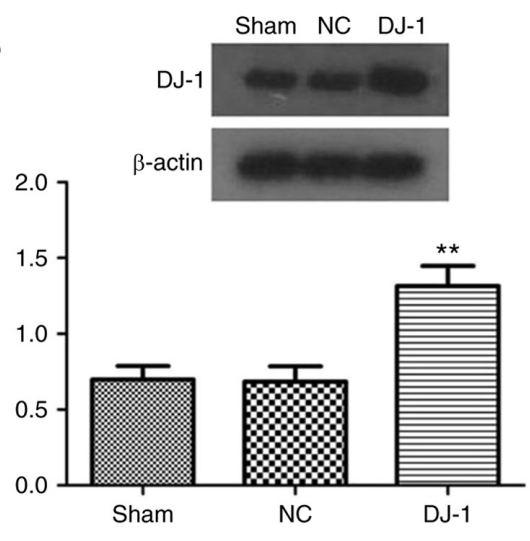

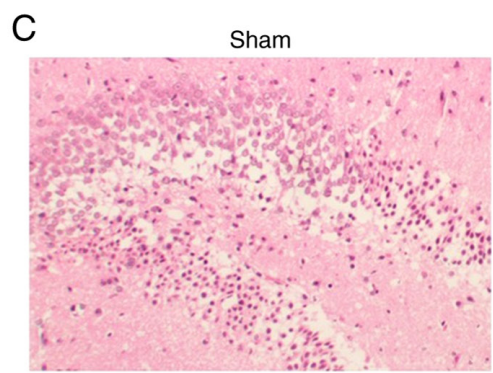

NC
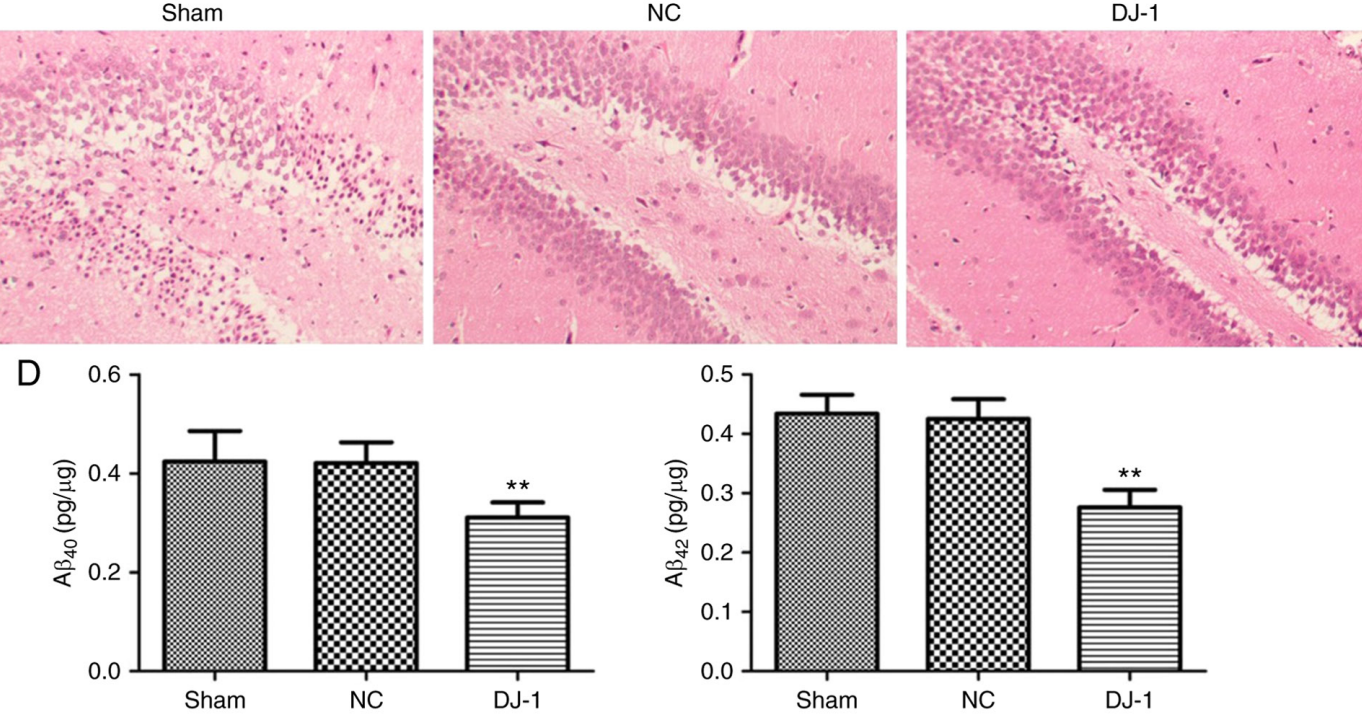

E
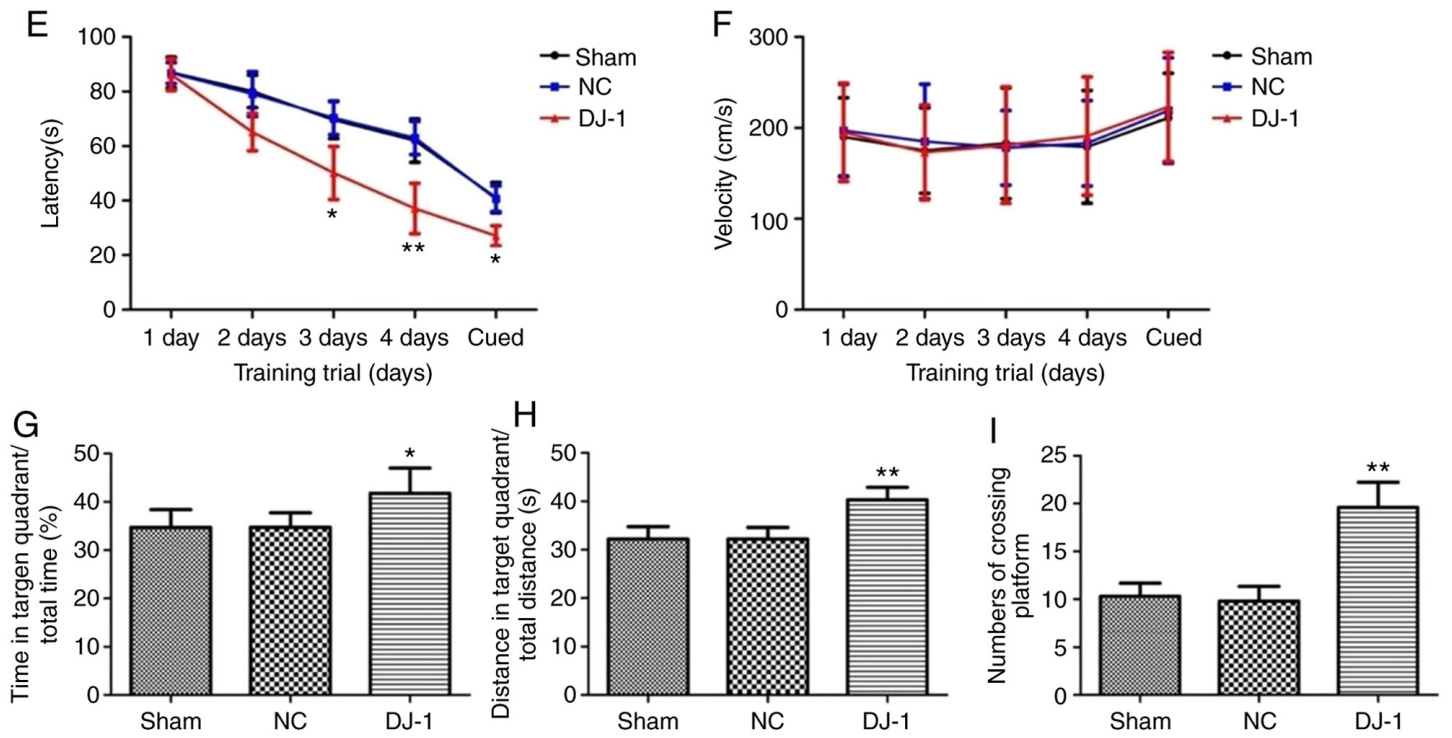

Figure 1. Effect of $D J-1$ overexpression on brain tissue damage and cognitive function in 5XFAD transgenic mice. The expression of $D J-1$ (A) mRNA and (B) protein in the hippocampus was detected by reverse transcription-quantitative PCR. (C) The pathological changes and neuron in hippocampus were detected by hematoxylin-eosin staining (magnification x400). (D) The content of $A \beta_{40}$ and A $\beta_{42}$ was measured by ELISA. (E and F) The cognitive function of 5XFAD mice was detected by the Morris water maze test. (E) During acquisition training, in the mice who underwent bilateral hippocampal injection of DJ-1 lentivirus reached the hidden platform in a shorter period than those in the sham group. (F) No difference existed in velocity between the two groups. Statistical data of $(\mathrm{G})$ the percent time that the mice spent in the target quadrant, $(\mathrm{H})$ distance traveled, and (I) the number of times that the mice crossed the platform area during the probe trial. $n=10$. Data are presented as the mean \pm standard deviation. ${ }^{*} \mathrm{P}<0.05$ and ${ }^{* * *} \mathrm{P}<0.01$ vs. sham group. $\mathrm{A} \beta, \beta$-amyloid protein; $\mathrm{NC}$, negative control.

the cytoplasm and increased the expression of Nrf2 in the nucleus (Fig. 4A). The results suggested that overexpression of $D J-1$ can promote the transfer of Nrf2 into the nucleus to exert an anti-oxidative stress effect. 
A

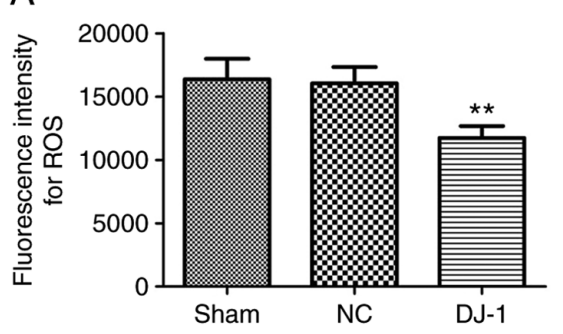

$\mathrm{B}$

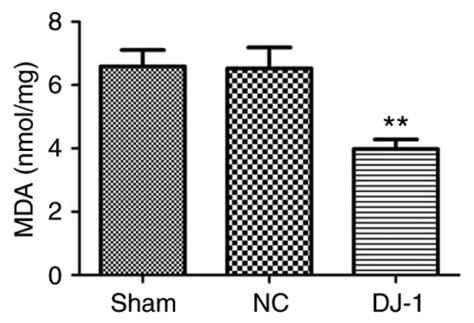

C

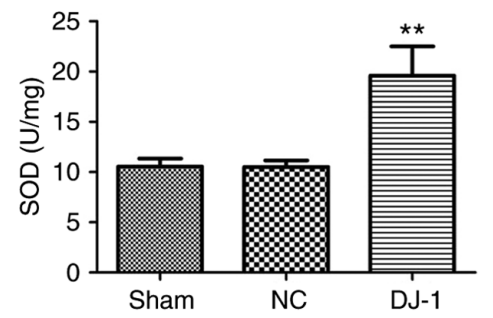

Figure 2. Effect of DJ-1 overexpression on oxidative stress in 5XFAD transgenic mice. Compared with the sham group, the (A) ROS activity and (B) MDA content in the hippocampus of mice with $D J-1$ lentivirus injected into the bilateral hippocampus were significantly decreased, while (C) the SOD activity was significantly increased. $\mathrm{n}=10$. Data are presented as the mean \pm standard deviation. ${ }^{* *} \mathrm{P}<0.01$ vs. sham group. ROS, reactive oxygen species; MDA, malondialdehyde; SOD, superoxide dismutase; NC, negative control.

A
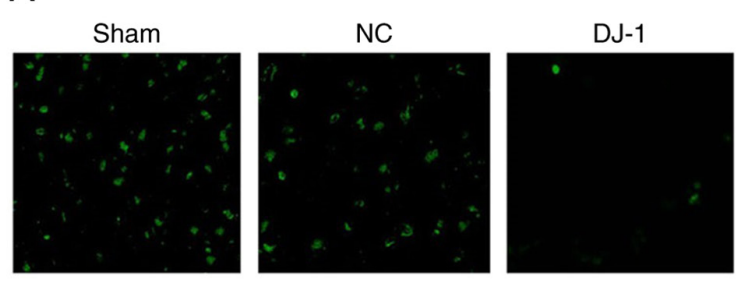

B

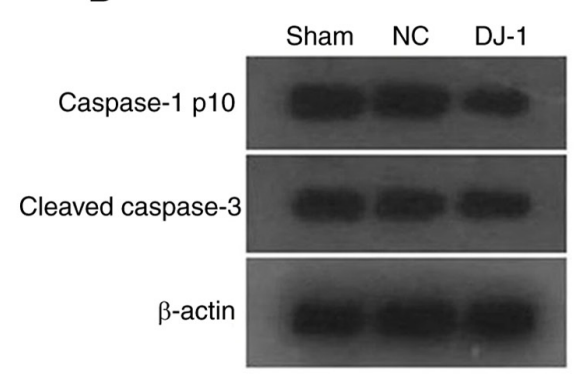

C

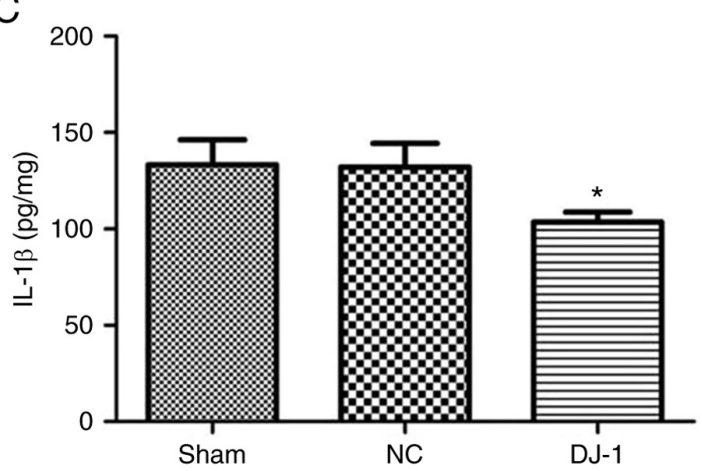

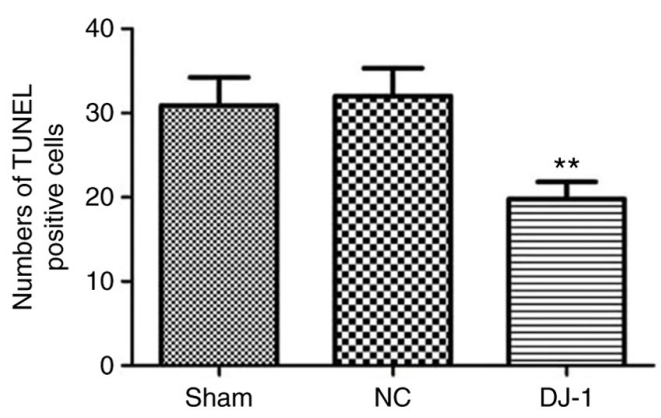

\& Caspase-1 p10

Cleaved caspase-3
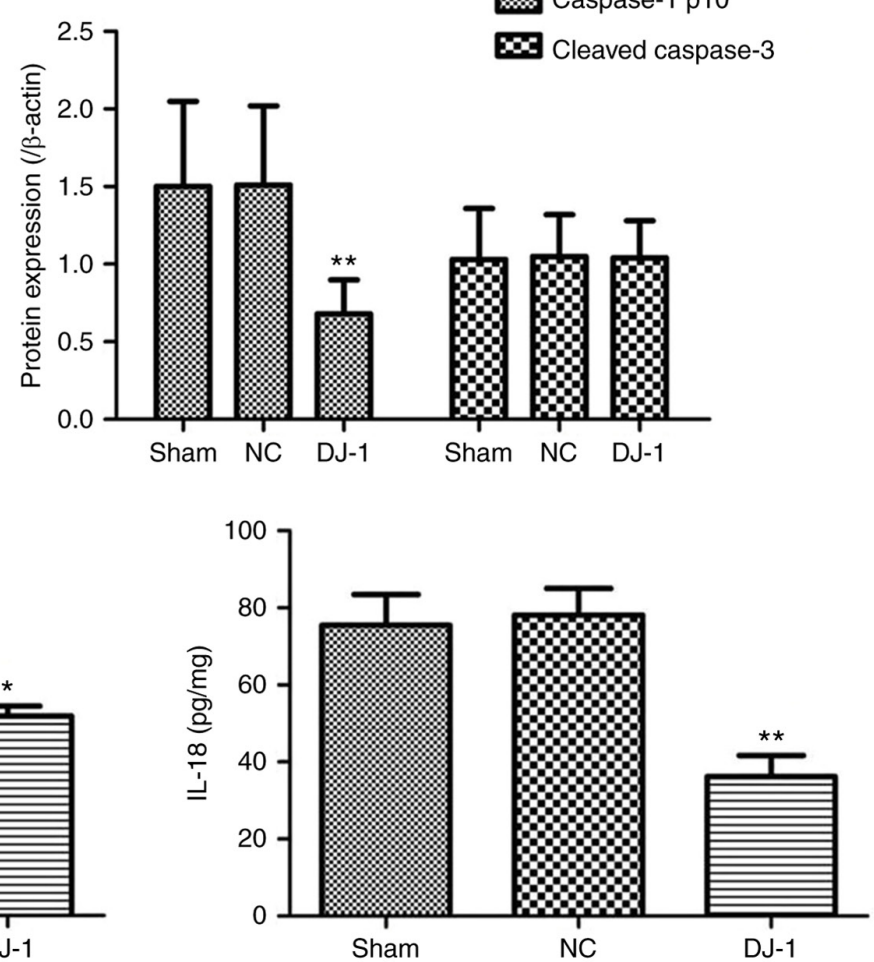

Figure 3. Effect of overexpression of $D J-1$ on neuronal pyroptosis in 5XFAD transgenic mice. (A) The neuronal apoptosis in hippocampus was detected by terminal transferase-mediated biotin dUTP nick end labeling assay (magnification x200). (B) The expression of caspase-1 and caspase-3 in hippocampus was measured by western blotting. (C) The levels of IL-1 $\beta$ and IL-18 were analyzed by ELISA. $n=10$. Data are presented as the mean \pm standard deviation. ${ }^{*} \mathrm{P}<0.05$ and ${ }^{* *} \mathrm{P}<0.01$ vs. sham group. IL, interleukin; NC, negative control.

A previous study has shown that the Nrf2 pathway serves an important role in the activation of NLRP3 inflammasome (35), while NLRP3 inflammasome activation is the most important complex protein formed during pyroptosis (7). It was identified that $D J-1$ overexpression in the brain significantly inhibited the expression of NLRP3 and ASC proteins in brain tissue 

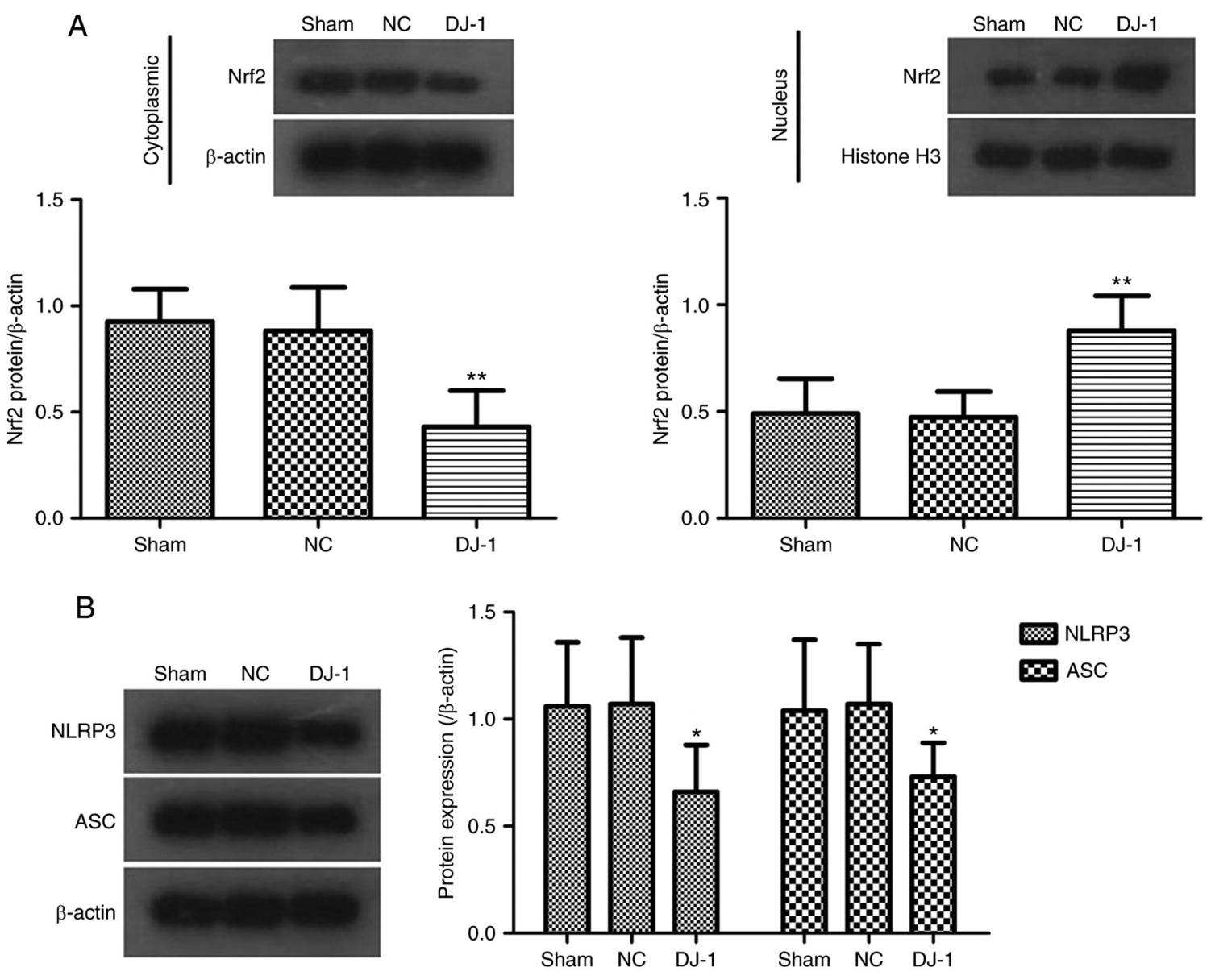

Figure 4. Effect of DJ-1 overexpression on expression of Nrf2-NLRP3-axis associated proteins in hippocampus of 5XFAD transgenic mice. (A) Western blotting and quantification of cytoplasmic and nuclear Nrf2 protein. $\beta$-actin and histone $\mathrm{H} 3$ were used for normalization for cytoplasmic and nuclear proteins, respectively. (B) Western blotting and quantification of NLRP3 and ASC protein expression. $n=10$. Data are presented as the mean \pm standard deviation ${ }^{*} \mathrm{P}<0.05,{ }^{* *} \mathrm{P}<0.001$ vs. sham group. Nrf2, nuclear factor erythroid 2-related factor 2; NLRP3, NLR pyrin domain containing 3; ASC, apoptosis-associated speck-like protein containing a CARD; NC, negative control.

(Fig. 4B), suggesting that $D J-1$ overexpression inhibits the activation of NLRP3 inflammasome.

\section{Discussion}

5XFAD transgenic mice, carrying 3 APP mutant genes and 2 PS1 mutant gene, have been used as AD model mice (36). The transgenic mice have been shown to have similar functional and pathological changes to AD. The most typical change in $\mathrm{AD}$ is the formation of senile plaques (SP) and the main cause of SP formation is the deposition of $A \beta$. As an important part of AD pathological changes, the hippocampus is closely associated learning and memory and cognitive function, and also participates in the regulation of the autonomic nervous system through the nuclei, endocrine system and neurotransmitters of the hypothalamus and brainstem (37). In the present study, it was identified that after overexpression of $D J-1$ in the brain, cognitive impairment, hippocampal tissue damage and $A \beta$ deposition were significantly improved in 5XFAD transgenic mice and the level of neuronal apoptosis was significantly decreased. These results suggested that $D J-1$ serves an important protective role in the development of AD.

Oxidative stress is an important pathological manifestation of AD. Decreased antioxidant capacity and increased oxidative stress products disrupt the dynamic balance of antioxidants and oxidation in the brain (5). SOD is an important antioxidant and MDA is an end product of cytotoxicity produced by lipid peroxidation. A previous study confirmed that abnormal changes in serum SOD and MDA have a higher sensitivity and specificity for predicting $\mathrm{AD}$ (38). Oxidative stress can induce mitochondrial function and damage of blood-brain barrier caused by cerebral vascular cell damage (39), whilst also cause clinical pathological symptoms of AD such as amyloid deposition, neurofibrillary tangles and cognitive dysfunction (40). Therefore, preventing oxidative stress has become an important strategy for the treatment of AD. $D J-1$ is considered to be an oxidative stress response protein (29) and is closely associated with the regulation of oxidative stress in nerve cells (30). Baulac et al (41) demonstrated that $D J-1$ is upregulated in the brain of patients with $\mathrm{AD}$ and is associated with elevated levels of oxidative stress. Mullett et al (42) suggested that $D J-1$ safeguards astrocyte-mediated protection against neuronal oxidative stress. Zhang et al (43) identified that the high expression of wild-type DJ-1 gene in SH-SY 5Y cells cultured in vitro has an inhibitory effect on oxidative damage, which may be associated with the decrease of intracellular ROS. Kim et al (44) reported that $D J-1^{-1-}$ embryonic cortical neurons showed increased sensitivity to oxidative stress and 
neurons overexpressing $D J-1$ were protected from oxidative stress in vitro. The present study identified that $D J-1$ overexpression in the brain of AD mice could increase SOD activity and decrease ROS activity and MDA content in the hippocampus, suggesting that $D J-1$ overexpression can decrease the degree of oxidative stress in AD mice. Therefore, it was hypothesized that $D J-1$ may inhibit A $\beta$ deposition by decreasing ROS activity, thereby lessening brain damage in AD mice. ROS increased the production of $\mathrm{A} \beta$ by promoting the expression of amyloid precursor protein and $\beta$-secretase. The production and aggregation of $A \beta$ further induces the formation of more oxygen free radicals, forming a vicious circle leading to neuronal degeneration and necrosis (24). Additionally, this positive feedback activity also promotes hyperphosphorylation of Tau protein and induces the accumulation of soluble Tau protein to form fibrils to promote the production of intracellular neurofibrillary tangles (NFTs) $(26,39)$. NFTs are also one of the characteristic pathological features of AD. However, whether $D J-1$ can affect the deposition of Tau by inhibiting oxidative stress requires further investigation.

Nrf2 is considered to be the main active factor of cellular responses to oxidative stress (45). As the most important endogenous antioxidant stress pathway identified at present, the Nrf2 pathway serves a key role in the development of AD (34). Under physiological conditions, Nrf2 binds to Kelch-like ECH-associated protein 1 (Keap1) to treat non-activated and rapidly degraded ubiquitin-proteasome (46). Under the stimuli of oxidative stress, the dissociation of Nrf2 and its inhibitory protein Keap1 inhibit the ubiquitination of Nrf2 to increase its stability. Stable Nrf2 is transferred to the nucleus and binds to an antioxidant response element (ARE) to initiate expression of a series of antioxidant proteins regulated by ARE (47). Clements et al (48) reported that DJ-1 serves a key role in the activation and regulation of the Nrf2 pathway. Sun et al (18) demonstrated that oxidative stress can activate $D J$-land its downstream Nrf2 pathway for endogenous protection in a diabetic rat model. High expression of $D J-1$ promotes the dissociation of Nrf2 from Keap1 and prevents the binding of Nrf2 to Keap1, and the ubiquitination of $\mathrm{Nrf} 2$, to restore the stability of $\mathrm{Nrf} 2$ against cellular oxidative stress and apoptosis damage (49). The present study also identified that overexpression of $D J-1$ in the brain of AD mice can significantly decrease the expression of Nrf2 protein in the cytosol and promote the entry of Nrf2 into the nucleus. It was hypothesized that overexpression of $D J-1$ in hippocampus of AD mice can effectively activate the Nrf2 signaling pathway to induce the expression of a series of antioxidant enzyme proteins to exert anti-oxidative stress.

Neuronal death is another important feature of AD. The death of nerve cells is regulated by a variety of factors, such as oxidative stress and inflammatory response. There are a number of methods of cell death, some of which are caspase-dependent (50). Caspase-1 and caspase-3 are important molecules that regulate cell death and apoptosis. Caspase- 3 is an important executive molecule of apoptosis. A previous study has shown that caspase-3 activation is the ultimate mediator of apoptosis in brain homogenates of dementia rats, which leads to the loss of neurons in AD model rats (31). Caspase-1 is primarily associated with cell death caused by inflammation (32). Bergsbaken et al (7) reported that host cells undergo caspase-1-dependent death from apoptosis when stimulated by pathogenic microorganisms or endogenous risk signals, called pyroptosis. Like apoptosis, pyroptosis is characterized by nuclear shrinkage, chromatin DNA fragmentation and TUNEL positive staining. Additionally, loss of cell membrane integrity and release of inflammatory cellular contents to induce an inflammatory response is an important feature of pyroptosis other than apoptosis (33). A large number of necrotic neurons in the hippocampus of AD mice and overexpression of $D J-1$ was observed to reduce the death of hippocampal neurons in AD mice. Simultaneously, it was identified that the overexpression of $D J-1$ could significantly decrease the expression of capsase-1 in hippocampus of AD mice, but had no significant effect on the expression of caspase-3. Further analysis indicated that overexpression of $D J-1$ significantly decreased the levels of IL-1 $\beta$ and IL-18 in the inflammatory mediators of hippocampus. Therefore, it was hypothesized that the improvement of $D J-1$ overexpression on cognitive function and brain damage in AD mice may involve caspase-1 mediated pyroptosis.

Inflammasome is an important complex protein formed during the activation of pyroptosis. NLRP3 inflammasome is currently the most widespread inflammasome, consisting of NLRP3, adaptor protein ASC and the effector protein pro-caspase-1. IL-1 $\beta$ and IL-18 are the end products of pyroptosis, which are formed by the activation of caspase-1 to cleave pro-IL-1 $\beta$ and pro-IL-18 (51). It is generally accepted that the neuroinflammatory response regulated by the pyroptosis key protein inflammasome and the neuronal loss caused by pyroptosis are associated with the pathogenesis of $\mathrm{AD}(52,53)$. The NLRP3 inflammasome may be involved in the promotion of A $\beta$ deposition by microglia (54). NLRP3 inhibitors can decrease $A \beta$ deposition and improve cognitive function in AD mice, while inhibiting pyroptosis by the activation of microglia and inflammasome in AD mice (55). NLRP3 knockout mice exhibit decreased levels of caspase-1 expression and decreased A $\beta$ aggregation, suggesting that NLRP3 is closely associated with the pathology of AD (56). To the best of our knowledge, the activation mechanism of NLRP3 has not yet been clarified. A previous study suggested that mitochondrial damage produces a large amount of ROS that activates NLRP3 inflammasome and produces an inflammatory response, suggesting that oxidative stress may be associated with pyroptosis and inflammatory response (57). Choi et al (58) observed severe mitochondrial oxidative damage and brain inflammatory response in APP/SP1 transgenic AD accompanied by activation of NLRP3 inflammasome. Notably, NLRP3 activation involves the production of ROS (59). A $\beta$ stimulates microglia to produce reactive oxygen species via mitochondria and NAD(P)H oxidase and also activates NLRP3 inflammasome to generate caspase-1 to increase the neurotoxicity of microglia (60). These studies suggest that oxidative stress may lead to neurosynaptic dysfunction and neuronal loss by activating the inflammasome-induced neuroinflammatory response. Simultaneously, ROS produced by oxidative stress may act as a signaling molecule to initiate pyroptosis to induce neuroinflammation and neuronal death (61). Therefore, anti-oxidation may inhibit the activation of inflammasome to decrease the production of pyroptosis. Previousstudies have confirmed that the Nrf2 pathway serves an important role in the activation of NLRP3 inflammatory bodies. Zhao et al (10) identified that Nrf2-deficient macrophages exhibited a decrease 
in the maturation and secretion of caspase- 1 and IL-1 $\beta$ and a decrease in ASC formation and suggested that $\mathrm{Nrf} 2$ is a necessary factor for the activation of NLRP3 inflammasome. However, Liu et al (62) reported that Nrf2 activation inhibits NLRP3 expression, caspase- 1 cleavage and subsequent IL-1 $\beta$ production, and suggested that the Nrf2 pathway serves a negative regulatory role in ROS-induced NLRP3 inflammatory body activation. Wang et al (20) identified that, in the AD mouse model, upregulation of Nrf 2 can inhibit the activation of NLRP3 inflammatory bodies by modulating the TXNIP pathway. The present study identified that $D J-1$ overexpression in the brain activates the Nrf2 pathway in the AD mouse model and also decreases the expression of NLRP3 and ASC in hippocampus. In combination with data from previous studies, we hypothesized that $D J-1$ may be involved in the protection of brain damage in AD mice by regulating Nrf2-NLRP3 axis to regulate oxidative stress and pyroptosis.

In summary, overexpression of $D J-1$ in the brain can improve the cognitive function and $\mathrm{A} \beta$ deposition of 5XFAD mice and serve an important protective role in the development of $\mathrm{AD}$. Its mechanism may be associated with the inhibition of oxidative stress and neuropyroptosis by regulating the Nrf2 signaling pathway. In pharmacological studies, stimulation of $D J-1$ by activators, which in turn regulates a variety of complex mechanisms including oxidative stress and cell coke, may lead to new breakthroughs in the prevention and improvement of AD.

\section{Acknowledgements}

Not applicable.

\section{Funding}

The authors were supported financially by Science and Technology Projects in Henan Province (grant no. 162102310258).

\section{Availability of data and material}

The datasets used and/or analyzed during the current study are available from the corresponding author on reasonable request.

\section{Authors' contributions}

LC designed the study, conducted the experiments and analyzed the data. WZ conducted most of the experiments and wrote the manuscript. Both authors read and approved the final manuscript.

\section{Ethics approval and consent to participate}

All experimental procedures were ethically approved by the Animal Use and Care Committee of the Nanyang Institute of Technology and were conducted in accordance with the National Institute for Health 'Guide for the Care and Use of Laboratory Animals' (23).

\section{Patient consent for publication}

Not applicable.

\section{Competing interests}

The authors declare that they have no competing interests.

\section{References}

1. Cummings J, Lee G, Mortsdorf T, Ritter A and Zhong K: Alzheimer's disease drug development pipeline: 2017. Alzheimer's Dement (N Y) 3: 367-384, 2017.

2. Graham WV, Bonito-Oliva A and Sakmar TP: Update on Alzheimer's disease therapy and prevention strategies. Annu Rev Med 68: 413-430, 2017.

3. Polettini J, Richardson LS and Menon R: Oxidative stress induces senescence and sterile inflammation in murine amniotic cavity. Placenta 63: 26-31, 2018.

4. Sankhla CS: Oxidative stress and Parkinson's disease. Neurol India 65: 269-270, 2017

5. Collin F, Cheignon $C$ and Hureau C: Oxidative stress as a biomarker for Alzheimer's disease. Biomark Med 12: 201-203, 2018.

6. Tan MS, Tan L, Jiang T, Zhu XC, Wang HF, Jia CD and Yu JT: Amyloid-Beta induces NLRP1-dependent neuronal pyroptosis in models of Alzheimer's disease. Cell Death Dis 2014: e1382, 2018.

7. Bergsbaken T, Fink SL and Cookson BT: Pyroptosis: Host cell death and inflammation. Nat Rev Microbiol 7: 99-109, 2009.

8. Hu Q, Zhang T, Yi L, Zhou X and Mi M: Dihydromyricetin inhibits NLRP3 inflammasome-dependent pyroptosis by activating the Nrf2 signaling pathway in vascular endothelial cells. Biofactors 44: 123-136, 2018.

9. Heo MJ, Kim TH, You JS, Blaya D, Sancho-Bru P and Kim SG: Alcohol dysregulates miR-148a in hepatocytes through FoxO1, facilitating pyroptosis via TXNIP overexpression. Gut 68: 708-720, 2019.

10. Zhao C, Gillette DD, Li X, Zhang Z and Wen H: Nuclear factor E2-related factor-2 (Nrf2) is required for NLRP3 and AIM2 inflammasome activation. J Biol Chem 289: 17020-17029, 2014.

11. Song L, Pei L, Yao S, Wu Y and Shang Y: NLRP3 inflammasome in neurological diseases, from functions to therapies. Front Cell Neurosci 11: 63, 2017.

12. Nagakubo D, Taira T,Kitaura H,Ikeda M, Tamai K, Iguchi-Ariga SM and Ariga H: DJ-1, a novel oncogene which transforms mouse NIH3T3 cells in cooperation with ras. Biochem Biophys Res Commun 231: 509-513, 1997.

13. McCoy MK and Cookson MR: DJ-1 regulation of mitochondrial function and autophagy through oxidative stress. Autophagy 7 : 531-532, 2011.

14. Xu J, Zhong N, Wang H, Elias JE, Kim CY, Woldman I, Pifl C, Gygi SP, Geula C and Yankner BA: The Parkinson's disease-associated DJ-1 protein is a transcriptional co-activator that protects against neuronal apoptosis. Hum Mol Genet 14: 1231-1241, 2005.

15. Gan L, Johnson DA and Johnson JA: Keap1-Nrf2 activation in the presence and absence of DJ-1. Eur J Neurosci 31: 967-977, 2010.

16. Wu L, Xu H, Cao L, Li T, Li R, Feng Y, Chen J and Ma J: Salidroside protects against mpp+-induced neuronal injury through DJ-1-Nrf2 antioxidant pathway. Evid Based Complem Alternat Med 2017: 5398542, 2017.

17. Sun Q, Shen ZY, Duan WN, Meng QT and Xia ZY: Mechanism of myocardial ischemia/reperfusion-induced acute kidney injury through DJ-1/Nrf2 pathway in diabetic rats. Exp Ther Med 14: 4201-4207, 2017.

18. Sun Q, Shen ZY, Meng QT, Liu HZ, Duan WN and Xia ZY: The role of DJ-1/Nrf2 pathway in the pathogenesis of diabetic nephropathy in rats. Ren Fail 38: 294-304, 2016.

19. Lev N, Barhum Y, Ben-Zur T, Aharony I, Trifonov L, Regev N, Melamed E, Gruzman A and Offen D: A DJ-1 based peptide attenuates dopaminergic degeneration in mice models of parkinson's disease via enhancing Nrf2. PLoS One 10: e0127549, 2015.

20. Wang CY, Xu Y, Wang X, Guo C, Wang T and Wang ZY: Dl-3-n-butylphthalide inhibits NLRP3 inflammasome and mitigates Alzheimer's-Like pathology via Nrf2-TXNIP-TrX axis. Antioxid Redox Signal 30: 1411-1431, 2019.

21. Hou Y, Wang Y, He Q, Li L, Xie H, Zhao Y and Zhao J: Nrf2 inhibits NLRP3 inflammasome activation through regulating Trx1/TXNIP complex in cerebral ischemia reperfusion injury. Behav Brain Res 336: 32-39, 2018.

22. Wree A, Eguchi A, McGeough MD, Pena CA, Johnson CD, Canbay A, Hoffman HM and Feldstein AE: NLRP3 inflammasome activation results in hepatocyte pyroptosis, liver inflammation, and fibrosis in mice. Hepatology 59: 898-910, 2014. 
23. Bayne K: Revised guide for the care and use of laboratory animals available. Physiologist 39: 208-211, 1996.

24. Lamberty Y, Gower AJ, Gobert J, Hanin I and Wulfert E: Behavioural, biochemical and histological effects of AF64A following injection into the third ventricle of the mouse. Behav Brain Res 51: 165-177, 1992.

25. Ahmad J, Baig MA, Ali AA, Al-Huqail A, Ibrahim MM and Qureshi MI: Comparative assessment of four RNA extraction methods and modification to obtain high-quality RNA from leaf. 3 Biotech 7: 373, 2017.

26. Livak KJ and Schmittgen TD: Analysis of relative gene expression data using real-time quantitative PCR and the 2(-Delta Delta C(T)) method. Methods 25: 402-408, 2001.

27. Vorhees CV and Williams MT: Morris water maze: Procedures for assessing spatial and related forms of learning and memory. Nat Protoc 1: 848-858, 2006.

28. Li HN, Jiang YM, Zhao P, Zhou S and Yu JQ: Effects of oxymatrine on oxidative stress in brain tissue of neonatal rats with hypoxic-ischemic brain damage. J Ningxia Med Uni 7: 743-745, 2016 (In Chinese).

29. Lakshminarasimhan M, Maldonado MT, Zhou W, Fink AL and Wilson MA: Structural impact of three Parkinsonism-associated missense mutations on human DJ-1. Biochemistry 47: 1381-1392, 2008.

30. Joselin AP, Hewitt SJ, Callaghan SM, Kim RH, Chung YH, Mak TW, Shen J, Slack RS and Park DS: ROS-dependent regulation of parkin and DJ-1 localization during oxidative stress in neurons. Hum Mol Genet 21: 4888-4903, 2012.

31. D'Amelio M, Cavallucci V, Middei S, Marchetti C, Pacioni S, Ferri A, Diamantini A, De Zio D, Carrara P, Battistini L, et al: Caspase-3 triggers early synaptic dysfunction in a mouse model of Alzheimer's disease. Nat Neurosci 14: 69-76, 2011.

32. Miao EA, Rajan JV and Aderem A: Caspase-1-induced pyroptotic cell death. Immunol Rev, 243: 206-14, 2011.

33. Fink SL and Cookson BT: Caspase-1-Dependent pore formation during pyroptosis leads to osmotic lysis of infected host macrophages. Cell Microbiol 8: 1812-1825, 2006.

34. Mota SI, Costa RO, Ferreira IL, Santana I, Caldeira GL, Padovano C, Fonseca AC, Baldeiras I, Cunha C, Letra L, et al: Oxidative stress involving changes in Nrf2 and ER stress in early stages of Alzheimer's disease. Biochim Biophys Acta 1852: $1428-1441,2015$

35. Liu X, Zhang X, Ding Y, Zhou W, Tao L, Lu P, Wang Y and $\mathrm{Hu}$ R: Nuclear factor E2-related factor-2 negatively regulates NLRP3 inflammasome activity by inhibiting reactive oxygen species-induced NLRP3 priming. Antioxidants Redox Signal 26 : 28-43, 2017.

36. Oakley H, Cole SL, Logan S, Maus E, Shao P, Craft J, Guillozet-Bongaarts A, Ohno M, Disterhoft J, Van Eldik L, et al: Intraneuronal beta-amyloid aggregates, neurodegeneration, and neuron loss in transgenic mice with five familial Alzheimer's disease mutations: Potential factors in amyloid plaque formation. J Neurosci 26: 10129-10140, 2006.

37. Liu WH, Shi LS, Chung MC, Chang TC and Lee SY: Antcamphin $M$ inhibits TLR4-mediated inflammatory responses by upregulating the Nrf2/HO-1 pathway and suppressing the nlrp3 inflammasome pathway in macrophages. Am J Chin Med 47: $1611-1626,2019$.

38. Lopez N, Tormo C, De Blas I, Llinares I and Alom J: Oxidative stress in Alzheimer's disease and mild cognitive impairment with high sensitivity and specificity. J Alzheimers Dis 33: 823-829, 2013.

39. Aliev G, Priyadarshini M, Reddy VP, Grieg NH, Kaminsky Y, Cacabelos R, Ashraf GM, Jabir NR, Kamal MA, Nikolenko VN, et al: Oxidative stress mediated mitochondrial and vascular lesions as markers in the pathogenesis of Alzheimer disease. Curr Med Chem 21: 2208-2217, 2014.

40. Bonda DJ, Wang X, Perry G, Nunomura A, Tabaton M, Zhu X and Smith MA: Oxidative stress in Alzheimer disease: A possibility for prevention. Neuropharmacology 59: 290-294, 2010.

41. Baulac S, Lu H, Strahle J, Yang T, Goldberg MS, Shen J, Schlossmacher MG, Lemere CA, Lu Q and Xia W: Increased DJ-1 expression under oxidative stress and in Alzheimer's disease brains. Mol Neurodegener 4: 12, 2009.

42. Mullett SJ, Di Maio R, Greenamyre JT and Hinkle DA: DJ-1 expression modulates astrocyte-mediated protection against neuronal oxidative stress. J Mol Neurosci 49: 507-511, 2013.
43. Zhang $\mathrm{Z}$, Wang $\mathrm{Q}$ and $\mathrm{Pu} \mathrm{XP}$ : Inhibition of oxidative damage by high expression of wild-type DJ-1 gene in SH-SY5Y cells cultured in vitro. Chin J New Drugs 16: 1854-1857, 2007 (In Chinese).

44. Kim RH, Smith PD, Aleyasin H, Hayley S, Mount MP, Pownall S, Wakeham A, You-Ten AJ, Kalia SK, Horne P, et al: Hypersensitivity of DJ-1-deficient mice to 1-methyl-4phenyl-1,2,3,6-tetrahydropyrindine (MPTP) and oxidative stress. Proc Natl Acad Sci USA 102: 5215-5220, 2005.

45. Yagishita Y, Uruno A, Fukutomi T, Saito R, Saigusa D, Pi J, Fukamizu A, Sugiyama F, Takahashi S and Yamamoto M: Nrf2 improves leptin and insulin resistance provoked by hypothalamic oxidative stress. Cell Rep 18: 2030-2044, 2017.

46. Kaspar JW, Niture SK and Jaiswal AK: Nrf2:INrf2 (Keap1) signaling in oxidative stress. Free Radic Biol Med 47: 1304-1309, 2009.

47. Nguyen T, Nioi P and Pickett CB: The Nrf2-antioxidant response element signaling pathway and its activation by oxidative stress. J Biol Chem 284: 13291-13295, 2009.

48. Clements CM, McNally RS, Conti BJ, Mak TW and Ting JP DJ-1, a cancer- and Parkinson's disease-associated protein, stabilizes the antioxidant transcriptional master regulator Nrf2. Proc Natl Acad Sci USA 103: 15091-15096, 2006.

49. Liu C, Chen Y, Kochevar IE and Jurkunas UV: Decreased DJ-1 leads to impaired Nrf2-regulated antioxidant defense and increased UV-A-induced apoptosis in corneal endothelial cells. Invest Ophthalmol Vis Sci 55: 5551-5560, 2014.

50. Zhang L, Zhang T and Tan N: Programmed cell death independent of caspases. Pro Mod Biomed 66: 3760-3763, 2009.

51. Liu D, Zeng X, Li X, Mehta JL and Wang X: Role of NLRP3 inflammasome in the pathogenesis of cardiovascular diseases. Basic Res Cardiol 113: 5, 2018.

52. Bossu P, Ciaramella A, Moro ML, Bellincampi L, Bernardini S, Federici G, Trequattrini A, Macciardi F, Spoletini I, Di Iulio F, et al: Interleukin 18 gene polymorphisms predict risk and outcome of Alzheimer's disease. J Neurol Neurosurg Psychiatry 78: 807-811, 2007.

53. Ojala J, Alafuzoff I, Herukka SK, van Groen T, Tanila H and Pirttila T: Expression of interleukin-18 is increased in the brains of Alzheimer's disease patients. Neurobiol Aging 30: 198-209, 2009.

54. Feng J, Wang JX, Du YH, Liu Y, Zhang W, Chen JF, Liu YJ, Zheng M, Wang KJ and He GQ: Dihydromyricetin inhibits microglial activation and neuroinflammation by suppressing NLRP3 inflammasome activation in APP/PS1 transgenic mice. CNS Neurosci Ther 24: 1207-1218, 2018.

55. Dempsey C, Rubio Araiz A, Bryson KJ, Finucane O, Larkin C, Mills EL, Robertson AAB, Cooper MA, O'Neill LAJ and Lynch MA: Inhibiting the NLRP3 inflammasome with MCC950 promotes non-phlogistic clearance of amyloid- $\beta$ and cognitive function in APP/PS1 mice. Brain Behav Immun 61: 306-316, 2017.

56. Heneka MT, Kummer MP, Stutz A, Delekate A, Schwartz S, Vieira-Saecker A, Griep A, Axt D, Remus A, Tzeng TC, et al: NLRP3 is activated in Alzheimer's disease and contributes to pathology in APP/PS1 mice. Nature 493: 674-678, 2013.

57. Sokolovska A, Becker CE, Ip WK, Rathinam VA, Brudner M, Paquette N, Tanne A, Vanaja SK, Moore KJ, Fitzgerald KA, et al: Activation of caspase-1 by the NLRP3 inflammasome regulates the NADPH oxidase NOX 2 to control phagosome function. Nat Immunol 14: 543-553, 2013.

58. Choi AJ and Ryter SW: Inflammasomes: Molecular regulation and implications for metabolic and cognitive diseases. Mol Cells 37: 441-448, 2014

59. Lu L, Lu Q, Chen W, Li J, Li C and Zheng Z: Vitamin D protects against diabetic retinopathy by inhibiting high-glucose-induced activation of the ros/txnip/nlrp3 inflammasome pathway. J Diabetes Res 22: 8193523, 2018.

60. Parajuli B, Sonobe Y, Horiuchi H, Takeuchi H, Mizuno T and Suzumura A: Oligomeric amyloid $\beta$ induces IL-1 $\beta$ processing via production of ROS: Implication in Alzheimer's disease. Cell Death Dis 4: e975, 2013.

61. Wali JA, Gurzov EN, Fynch S, Elkerbout L, Kay TW, Masters SL and Thomas HE: Activation of the NLRP3 inflammasome complex is not required for stress-induced death of pancreatic islets. PLoS One 9: e113128, 2014.

62. Liu X et al: Nuclear Factor E2-Related Factor-2 Negatively Regulates NLRP3 Inflammasome Activity by Inhibiting Reactive Oxygen Species-Induced NLRP3 Priming.[J]. Antioxid Redox Signal 26:28, 2017.

This work is licensed under a Creative Commons Attribution-NonCommercial-NoDerivatives 4.0 International (CC BY-NC-ND 4.0) License. 